OPEN ACCESS

Edited by:

Pamela Ann McCombe,

The University of

Queensland, Australia

Reviewed by:

Ruben Van Eijk,

University Medical Center

Utrecht, Netherlands

Mamede De Carvalho,

University of Lisbon, Portugal

Thomas Shaw

The University of

Queensland, Australia

Christopher A. Olm,

University of Pennsylvania,

United States

*Correspondence:

Jie Lu

imaginglu@hotmail.com

Specialty section: This article was submitted to Movement Disorders, a section of the journal

Frontiers in Neurology

Received: 08 October 2021

Accepted: 12 January 2022

Published: 24 February 2022

Citation:

Li Q, Zhu W, Wen X, Zang Z, Da Y and Lu J (2022) Beyond the Motor Cortex:

Thalamic Iron Deposition Accounts for Disease Severity in Amyotrophic Lateral Sclerosis.

Front. Neurol. 13:791300. doi: 10.3389/fneur.2022.791300

\section{Beyond the Motor Cortex: Thalamic Iron Deposition Accounts for Disease Severity in Amyotrophic Lateral Sclerosis}

\author{
Qianwen $\mathrm{Li}^{1,2}$, Wenjia Zhu ${ }^{3}$, Xinmei Wen ${ }^{3}$, Zhenxiang Zang ${ }^{1,2}$, Yuwei $\mathrm{Da}^{3}$ and Jie $L u^{1,2 *}$ \\ ${ }^{1}$ Department of Radiology and Nuclear Medicine, Xuanwu Hospital, Capital Medical University, Beijing, China, ${ }^{2}$ Beijing Key \\ Laboratory of Magnetic Resonance Imaging and Brain Informatics, Beijing, China, ${ }^{3}$ Department of Neurology, Xuanwu \\ Hospital, Capital Medical University, Beijing, China
}

Objective: Previous studies have reliably identified iron deposition in the motor cortex as potential pathogenesis of amyotrophic lateral sclerosis (ALS). Here, we intended to investigate iron deposition, gray matter (GM) atrophy, and their associations with disease severity in the motor cortex and the thalamus in patients with ALS.

Methods: A total of 34 patients with ALS (age $51.31 \pm 8.24$ years, 23 males) and 34 nonneurological controls (age $50.96 \pm 9.35$ years, 19 males) were enrolled between 2018 and 2020. The Revised ALS Functional Rating Scale (ALSFRS-R) and the Penn upper motor neuron (UMN) score were measured. MRI data included quantitative susceptibility mapping (QSM) for iron deposition and three-dimensional (3D) T1 for gray matter volume. After a between-group comparison, Pearson's correlation coefficient was used for identifying correlations of iron deposition, GM volume, and clinical measurements.

Results: The two-sample $t$-tests revealed increased iron deposition in the left precentral gyrus (peak voxel $T=4.78, P_{\text {SvC }}=0.03$ ) and the thalamus (peak voxel: right: $T=6.38$, $P_{\text {SVC }}<0.001$; left: $\left.T=4.64, P_{\text {SVC }}=0.02\right)$ in patients with ALS. GM volume of the precentral gyrus $(T=-2.42, P=0.02)$ and the bilateral thalamus $(T=-4.10$, $P<0.001)$ were reduced. Negative correlations were found between the increased QSM values and the decreased GM volume $(P<0.04$, one-tailed) in patients with ALS. Iron deposition in the left precentral gyrus was positively correlated with the UMN score $(R=0.40, P=0.02)$ and the GM volume was negatively correlated with the UMN score $(R=-0.48, P=0.004)$. Negative correlation between thalamic iron deposition and the ALSFRS-R $(R=-0.36, P=0.04)$ score was observed.

Discussion: Iron deposition in the thalamus, in addition to the motor cortex, is accompanied by GM atrophy and is associated with disease severity in patients with ALS, indicating that the thalamus is also a pathological region in patients with ALS.

Keywords: amyotrophic lateral sclerosis (ALS), iron deposition, gray matter atrophy, motor cortex, thalamus 


\section{BACKGROUND}

Amyotrophic lateral sclerosis (ALS) is a progressive neurodegenerative disorder characterized by impairments of motor neurons in the motor cortex, brainstem, and spinal cord (1). Nowadays, increasing observations of extramotor impairments were reported in ALS studies $(2,3)$. Particularly, as one of the major subcortical areas that modulate information via the thalamocortical loop, the thalamus is critical for the investigation of the pathological mechanism of $\operatorname{ALS}(4,5)$.

Studies over the past decades have shown gray matter (GM) atrophy in the motor cortex and the thalamus in patients with ALS $(6,7)$. Patients with ALS exhibited GM volume reduction in the motor cortex and the thalamus as shown in a longitudinal study (8). In a recent large cohort MRI-based study, Chipika and colleagues have shown atrophy of thalamic nuclei in both the chromosome 9 open reading frame 72 (C9orf72) carrier and noncarrier patients with ALS, demonstrating thalamus as a potential pathological area in the brain (9).

Furthermore, accumulating evidence suggested that iron deposition in the brain might have an important role in the pathogenesis of ALS $(10,11)$. A large amount of invivo MRI studies had reported iron deposition in the motor cortex $(10,12,13)$, which was in line with the postmortem study (10). Further, iron deposition in the brain was positively associated with the upper motor neuron (UMN) score of the corresponding limb (13). While postmortem examination (14) had shown iron deposition in the thalamus, in-vivo evidence showing how iron content differentiates between patients with ALS and nonneurological controls (NCs) in the thalamus is still lacking. Besides, little, if not nothing, is known regarding the association between thalamus iron deposition, GM atrophy, and clinical measurements.

On the cellular and molecular level, excessive iron deposition may lead to oxidative stress-associated neural apoptosis (15). On the macroscopic scale, cortical iron deposition in the precentral gyrus was correlated with GM atrophy in patients with multiple sclerosis (16). Suggestively, therefore, we hypothesize that the accumulation of iron content would accompany progressive GM atrophy in the motor cortex and the thalamus in patients with ALS. Investigation of their associations may provide new insights for underlying the mechanism of ALS.

Conclusively, we have two aims in this study. First, we intended to assess iron deposition and GM atrophy in the motor cortex and the thalamus in patients with ALS by using quantitative susceptibility mapping (QSM) and threedimensional (3D) T1 structural MRI modalities. Second, we intended to investigate how iron deposition and GM atrophy correlate with clinical neurological measurements.

\section{MATERIALS AND METHODS}

\section{Study Participants}

This study was approved by the Ethic Committees of Xuanwu Hospital, Capital Medical University. We collected 40 patients with ALS and matched 42 non-NCs among volunteers and nonblood relatives between December 2018 and January 2020.
Patients with ALS were defined as "probable" or "definite" according to the revised El Escorial criteria (17). The NCs were recruited in Xuanwu Hospital with similar age and sex with the ALS patients. The controls did not show any signs of movement problem during the interview. Both the patients with ALS and the NCs were considered cognitively normal via the neurologists during the interview. All the participants were free of substance drug abuse, traumatic brain injury, cerebrovascular events, neuroinflammation, neoplastic conditions, and were able to participate in MRI scanning (e.g., without metallic plantin devices and severe disability). After the MRI scanning, one patient with ALS was excluded due to claustrophobia, and three patients with ALS and two controls were excluded due to severe motion artifacts. No space-occupying lesions were found in patients.

We recorded clinical characteristics, including the disease duration (DD) (number of months from the ALS symptoms onset to the MRI examination), the Revised ALS Functional Rating Scale (ALSFRS-R) (18), and the Penn UMN score (19). We then defined the disease progression rate (DPR) delta-FRS, which was calculated by using the formula: $\mathrm{DPR}=(48-\mathrm{ALSFRS}-\mathrm{R}) / \mathrm{DD}(20)$.

\section{Image Acquisition}

The imaging data were collected on a $3 \mathrm{~T}$ scanner (MR750, GE Healthcare Medical Systems, Chicago, Illinois, USA) equipped with an 8-channel head coil at the Xuanwu Hospital, Capital Medical University, Beijing, China. The standard MRI protocol included a multi-echo gradient echo (GRE) sequence and one 3D T1-weighted acquisition [3D brain volume imaging (BRAVO)]. The MR protocol is shown as below: GRE acquisition parameters: 12 echoes with $\mathrm{TE} 1=2.1 \mathrm{~ms}$ and $\Delta$ echo time $(\mathrm{TE})=2.4 \mathrm{~ms}$, repetition time $(\mathrm{TR})=31.1 \mathrm{~ms}$, flip angle $=20^{\circ}, \mathrm{FOV}=256 \mathrm{~mm}$ $\times 256 \mathrm{~mm}$, slice thickness $=1 \mathrm{~mm}$, matrix $=256 \times 256$, and 6.5min acquisition time. 3D BRAVO acquisition parameters: $\mathrm{TR}=$ $6.66 \mathrm{~ms}, \mathrm{TE}=2.93 \mathrm{~ms}$, inversion time $(\mathrm{TI})=450 \mathrm{~ms}$, flip angle $=$ $12^{\circ}$, field of view $(\mathrm{FOV})=256 \mathrm{~mm} \times 256 \mathrm{~mm}$, slice thickness $=$ $1 \mathrm{~mm}$, matrix $=256 \times 256$, and 4 -min acquisition time.

\section{Image Processing}

\section{Quantitative Susceptibility Mapping}

Quantitative susceptibility mapping is a technique that calculates the quantitative magnetic susceptibility values of tissues by deconvolving MRI phase signals with models of magnetic susceptibility.

The reconstruction of susceptibility MRI was performed by using STI suite version 3.0 (https://people.eecs.berkeley.edu/ chunlei.liu/software.html) as described in the previous study (21). In summary, phase image was combined with magnitude for new phase calculation. Then, the magnitude images were used for segmentation by using the brain extraction tool (BET) in FSL to generate a brain mask (22). The new phase images were then unwrapped with the Laplacian method (23) and the background phase was removed by using the variable-kernel sophisticated harmonic artifact reduction for phase data (V-SHARP) filtering method. Finally, the susceptibility map was generated by using the STQR-QSM (streaking artifacts reduction QSM) algorithm 
(24). The susceptibility map contains the QSM values [parts per million ( $\mathrm{ppm})$ ] in each voxel, which correlated strongly with the chemically determined iron concentration in the GM structures as shown in a previous postmortem study (25).

For spatial normalization purposes, we first registered the individual susceptibility maps onto the 3D T1 images. Next, the T1 images were segmented into six tissue maps and normalized by using Diffeomorphic Anatomical Registration Through Exponentiated Lie Algebra (DARTEL) algorithm (26) into the standard Montreal Neurological Institute (MNI) template. The deformation field was generated as the normalization parameters for the T1 images, which was later directly applied to the coregistered individual susceptibility maps. Images from eight participants were excluded due to inaccurate normalization (mismatch between template and QSM image of an individual, shown in Supplementary Figure S1 as an example), leaving 34 patients with ALS and 34 NCs into group analyses.

\section{Voxel-Based Morphometry (VBM) Analysis}

T1 data were analyzed by using the standard VBM approach to calculate the GM volume. In detail, T1-weighted structural images were preprocessed by using the CAT12 toolbox (http:// www.neuro.uni-jena.de/cat/). All the structural images were segmented into GM, white matter (WM), and cerebrospinal fluid (CSF). The GM images were normalized to the MNI space by using high-dimensional DARTEL normalization. Images were modulated via the Jacobian transformation to ensure that relative GM volumes were well preserved following spatial normalization. Prior to statistical analysis, the normalized modulated GM images were smoothed by using a $4-\mathrm{mm}$ full width at half maximum (FWHM) Gaussian kernel.

\section{Statistical Analysis}

Comparison of QSM between the ALS and control group was conducted in SPM12 in a voxel-wise manner. We used small volume correction (SVC) to account for multiple comparison issues within the precentral gyrus and the thalamus. Clusters with (1) peak voxel surviving $P<0.05$ SVC within the corresponding brain area $[20 \mathrm{~mm}$ sphere around the peak voxel overlapped with the corresponding automated anatomical labeling (AAL) regions (27)] and (2) with over $100 \mathrm{~mm}^{3}$ (100 voxels) surviving an arbitrary threshold $(P<0.005$ uncorrected $)$ were considered significant. We used the latter arbitrary criteria to ensure that the size of the significant clusters was not too small.

The regions that showed significant changes of QSM value were further used as a region of interest (ROIs) for extracting the mean GM volumes of brain structures including the precentral gyrus and the thalamus. After the QSM comparison, the GM volumes of the precentral gyrus and the thalamus were extracted by using the clusters that showed an increased iron deposition. We used SPSS software (IBM SPSS Statistics, version 25.0.0.1, IBM Incorporation, Armonk, New York, USA) for post-hoc analyses including comparisons of GM volume, associations with the QSM values as well as clinical measurements. The Pearson's correlation coefficient was used to assess the correlation between the QSM value and GM volume, QSM value, GM volume, and their associations with clinical measurements. Sex
TABLE 1 | The demographic and clinical profile of study participants.

\begin{tabular}{lcll}
\hline Characteristic & ALS patients & $\begin{array}{l}\text { Non-neurological } \\
\text { controls }\end{array}$ & P values \\
\hline Number & $34(40$ recruited) & $34(42$ recruited) & \\
Age & $51.31 \pm 8.24(\mathrm{SD})$ & $50.96 \pm 9.35(\mathrm{SD})$ & 0.56 \\
Sex (male) & $23(67.6 \%)$ & $19(55.9 \%)$ & 0.32 \\
ALSFRS-R & $40.06 \pm 4.15(\mathrm{SD}),[29-45]$ & NA & \\
DD (month) & $16.72 \pm 15.77(\mathrm{SD}),[2-84]$ & NA & \\
DPR (delftaFRS) & $0.9 \pm 0.95(\mathrm{SD}),[0.04-3.75]$ & NA \\
UMN score & $9.88 \pm 6.04$ (SD), [0-16] & NA &
\end{tabular}

ALSFRS-R, Revised Amyotrophic Lateral Sclerosis Functional Rating Scale; DD, disease duration; DPR, disease progression rate; UMN score, upper motor neuron score.

difference between the ALS and NCs groups was assessed with the chi-squared test. For analyses conducted in SPSS, results were considered significant if $P<0.05$.

\section{RESULTS}

\section{Clinical Data}

A total of 34 patients with ALS and 34 age- and sex-matched NCs were finally included in our analyses. Five of the patients with ALS were bulbar onset. Detailed demographic information of all the subjects is shown in Table 1. There were no significant differences in age $(P=0.56)$ and sex $(P=0.32)$ between the two groups.

\section{Imaging Data Increased Iron Deposition in the Left Precentral Gyrus and the Bilateral Thalamus}

We focused only on the precentral gyrus and the thalamus. Compared with NCs, the two-sample $t$-test revealed the significant increased QSM values in the left precentral gyrus (peak MNI coordinate: $\mathrm{x}=-40, \mathrm{y}=-24, \mathrm{z}=59$, peak $T$ value $=4.78, P_{\mathrm{SVC}}=0.03,220$ voxels with $\left.P_{\text {uncorrected }}<0.005\right)$ and the thalamus (right: peak MNI coordinate: $\mathrm{x}=20, \mathrm{y}=-17, \mathrm{z}=7$, peak $\mathrm{T}$ value $=6.38, P_{\mathrm{SVC}}<0.001$; left: peak MNI coordinate: $\mathrm{x}$ $=-13, \mathrm{y}=-5, \mathrm{z}=7$, peak $\mathrm{T}$ value $=4.64, P_{\mathrm{SVC}}=0.02,1,674$ voxels with $P_{\text {uncorrected }}<0.005$ ) in patients with ALS (Figure 1). No significant increased QSM values were found in the right precentral gyrus ( 82 voxels survived $P<0.005$ uncorrected threshold). The peak voxel did not reach small volume correction significance, with $P_{\text {corrected }}>0.9, T=3.89$ ). The $\mathrm{QSM}$ value of the two groups was normally distributed (Lilliefors test, $P>$ 0.31 ). Whole-brain increasing QSM distribution was given in Supplementary Figure S2.

\section{Gray Matter Atrophy in the Left Precentral Gyrus and the Bilateral Thalamus}

Mean GM volume of the left precentral gyrus and the bilateral thalamus was extracted from the clusters shown in Figure 1 ( $\left.P_{\text {uncorrected }}<0.005\right)$. Decreased GM volume was found in the precentral gyrus $(T=-2.42, P=0.02)$ and the bilateral thalamus $(T=-4.10, P<0.001)$ in patients with ALS (Figure 2). 
A
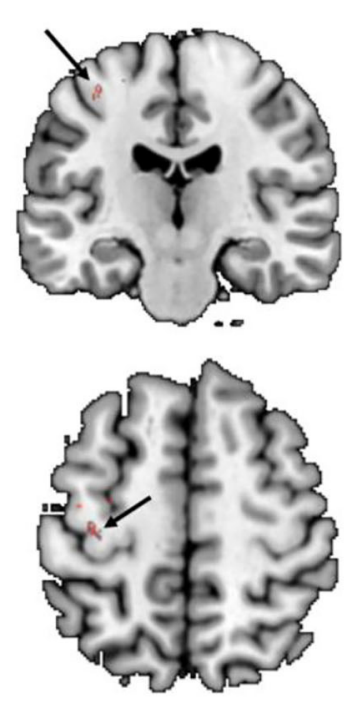

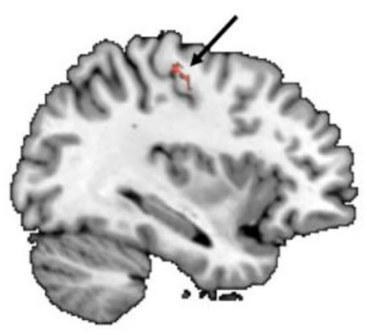

4.78

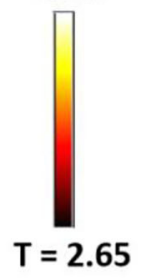

B
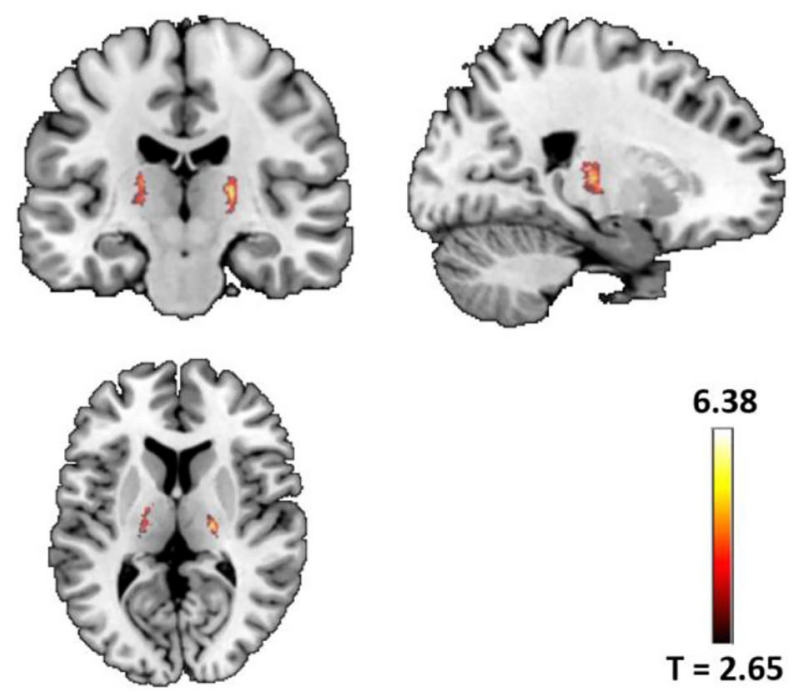

6.38

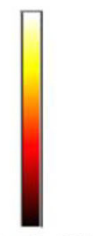

$\mathrm{T}=\mathbf{2 . 6 5}$

FIGURE 1 | (A) Significant increased quantitative susceptibility mapping (QSM) values were found in the left precentral gyrus (arrow) in patients with amyotrophic lateral sclerosis (ALS) (peak voxel $P_{\text {SVC }}=0.03$ ). (B) Significant increased QSM values were found in the bilateral thalamus in patients with ALS (left $P_{\text {SvC }}=0.02$; right $\left.P_{\text {SVC }}<0.001\right)$. Images were shown with a $P<0.005$ uncorrected thresholds and masked with the left precentral and the thalamus from the AAL atlas. In total, there were 220 voxels and 1,674 voxels surviving $P_{\text {uncorrected }}<0.005$ thresholds in the left precentral gyrus and the bilateral thalamus, respectively.

Total intracranial volume (TIV) was controlled as a covariate of noninterest. The GM volume of the two groups was normally distributed (Lilliefors test, $P>0.14$ ). Whole-brain GM reduction distribution was given in Supplementary Figure S3.

\section{Association Between Iron Deposition and GM Atrophy}

Since patients with ALS simultaneously exhibited increased iron deposition and decreased GM volume, we, therefore, specifically investigated the negative correlation between the two phenotypes. As shown in the results, the QSM values were significantly negatively correlated with the GM volume. In details, the QSM values were negatively correlated with GM volume in the left precentral gyrus $(R=-0.44, P=0.004$, onetailed) and the bilateral thalamus $(R=-0.32, P=0.04$, onetailed) in patients with ALS (Figure 3). The QSM values were neither correlated with GM volume in the left precentral gyrus ( $R=-0.17, P>0.16$, one-tailed) nor in the bilateral thalamus $(R$ $=-0.19, P>0.14$, one-tailed) in the control group.

\section{Clinical Associations}

Quantitative susceptibility mapping value of the left precentral gyrus was significantly positively correlated with the UMN score $(R=0.40, P=0.02)$ and the QSM value of the bilateral thalamus was significantly negatively correlated with the ALSFRS-R $(R$ $=-0.36, P=0.04$ ) (Figures 4A,B). GM volume of the left precentral gyrus was negatively correlated with the UMN score ( $R=-0.48, P=0.004$ ) (Figure 4C). No other significant correlations were found between the QSM value, GM volume, and other clinical characteristics (including DD, ALSFRS-R, and the DPR, all $P>0.15$ ).

\section{Age and Sex Influence}

We performed correlation analyses on age and QSM/GM values in both the patients and control group and found no significant correlations in any group (lowest $P=0.31$ ). We then controlled age as a covariate of noninterest and still found significant correlations between QSM and GM ( $P=0.004$ for precentral gyrus and $P=0.028$ for thalamus, one-tailed). In addition, there were no significant differences between male and female on QSM/GM values (lowest $P=0.33$ ) for either group.

\section{DISCUSSION}

Our primary goal was to investigate iron deposition and GM atrophy as well as their association in the motor cortex and thalamus in patients with ALS. We found an increased iron deposition and decreased GM volume in the motor cortex and the thalamus via QSM and VBM approaches, respectively. In patients with ALS, the increased iron deposition was negatively correlated with GM volume. Furthermore, iron deposition in the motor cortex was positively correlated with the UMN score, while iron deposition in the thalamus was negatively correlated with the ALSFRS-R score.

Since impairments of the motor cortex are the hallmark feature, most ALS imaging studies focus on evaluating structural and functional alterations of this region. As a result, GM volume reduction and iron deposition in the motor cortex have been consistently identified and replicated $(6,7,10)$. In this study, as 
A

\section{Left Precentral Gyrus}

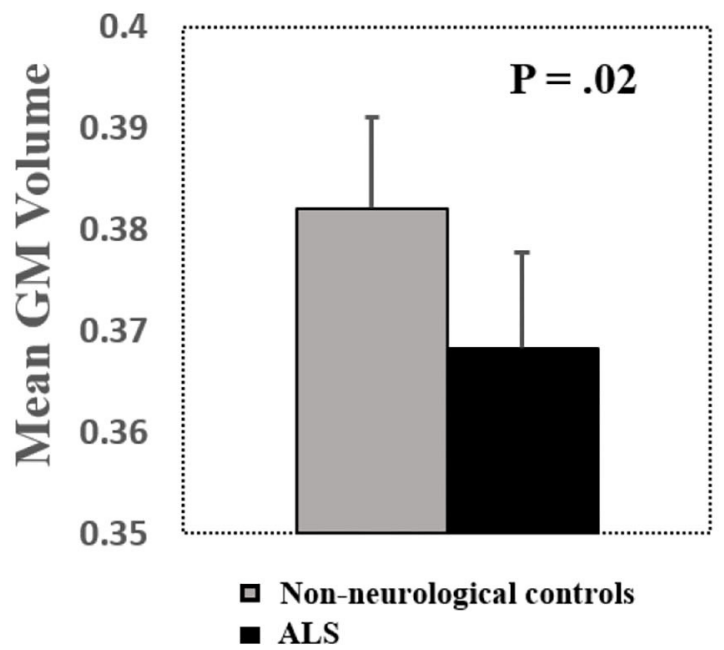

\section{Bilateral Thalamus}

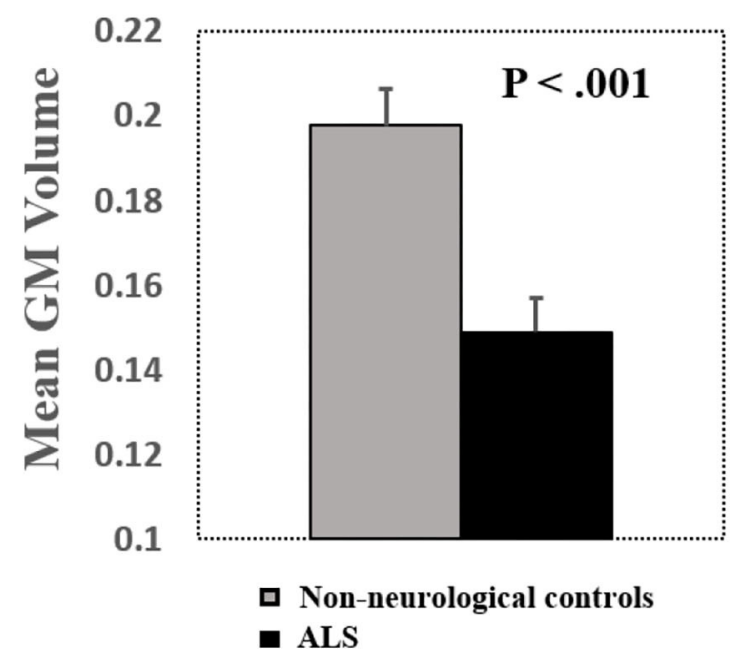

FIGURE 2 | (A) Significant brain volume atrophy was found in the left precentral gyrus in patients with ALS. (B) Significant brain volume atrophy was found in the bilateral thalamus in patients with ALS. Bar plots show mean gray matter (GM) volume after controlling for total intracranial volume (TIV) as a covariate of noninterest. Error bars show SEs.

A

\section{Left precentral Gyrus}

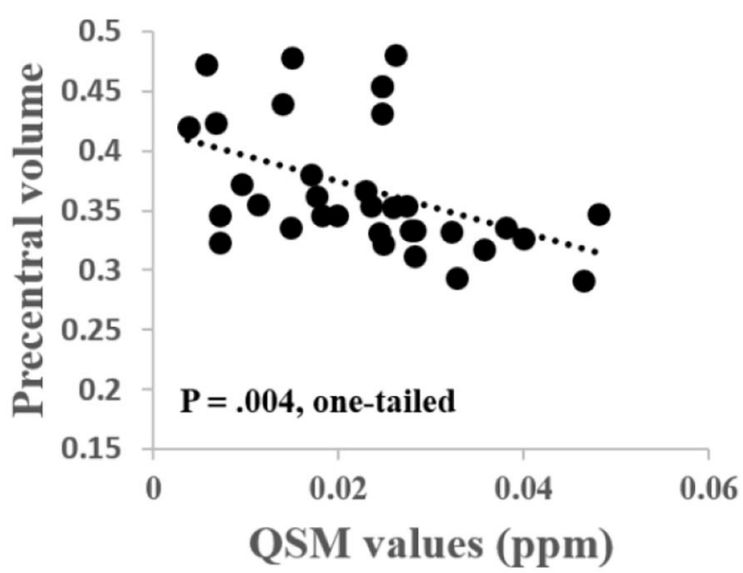

B

\section{Bilateral Thalamus}

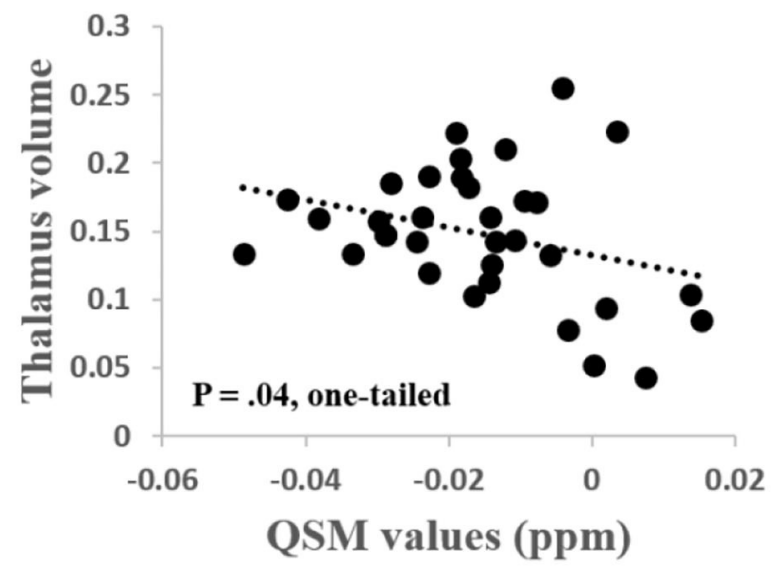

FIGURE 3 | (A) Significant negative correlation was found between the QSM values and GM volume in the left precentral gyrus $(R=-0.44, P=0.004$, one-tailed) and (B) Bilateral thalamus $(R=-0.32, P=0.04$, one-tailed) in patients with ALS.

expected, we detected a higher QSM value in a cluster adjacent to the "hand-knob" of the left precentral gyrus. We, in addition, found decreased GM volume in the same cluster. Similarly, we detected increased QSM value and decreased GM volume that largely overlapped with the ventral lateral and posterior nucleuses of the thalamus-areas that were functionally connected with the motor and somatosensory cortex $(28,29)$. Higher QSM value in the thalamus was highly in line with a postmortem study that revealed iron deposition in the thalamus (14). The increase of iron deposition in the brain is consistent with the observations from postmortem studies that reported intraneuronal TAR DNA-binding protein-43 (TDP-43) immunoreactivity and ferritin-positive microglia and macrophages. That being said, increased QSM in patients with ALS may indicate oxidative 
A

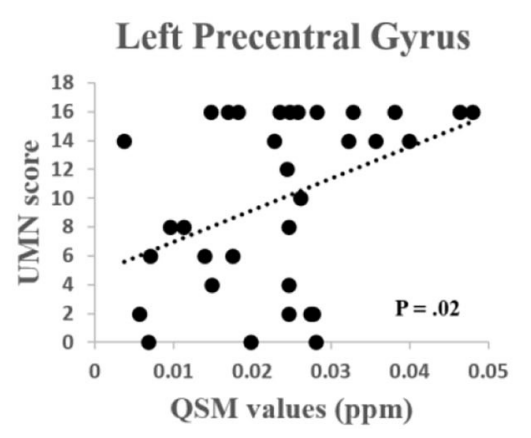

B

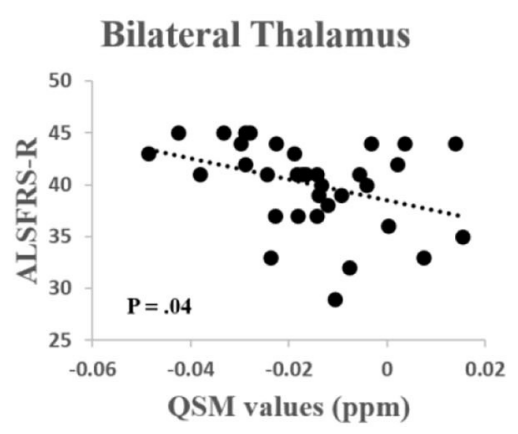

C

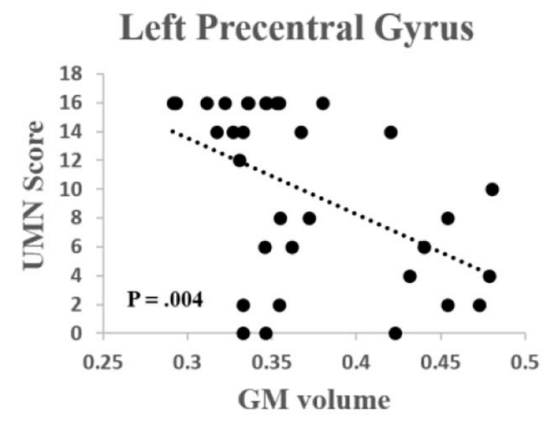

FIGURE 4 | (A) A significant positive correlation was found between the QSM values in the left precentral gyrus and the upper motor neuron (UMN) score $(R=0.40, P$ $=0.02)$. (B) A significant negative correlation was found between the QSM values in the thalamus and the Revised ALS Functional Rating Scale (ALSFRS-R) score ( $R$ $=-0.36, P=0.04)$. (C) A significant negative correlation was found between the GM volume in the left precentral gyrus and the UMN score $(R=-0.48, P=0.004)$.

stress-induced cell death and, possibly, phosphorylated $43 \mathrm{kDa}$ TAR DNA-binding protein (pTDP-43) accumulation (30). Our results support the idea of motor cortical and thalamic iron accumulation as pathological phenotypes in patients with ALS.

Increased iron deposition may result in the production of toxic free radicals, which can subsequently cause oxidative stress to neurons that may attenuate neuronal function $(15,31,32)$. Indicatively, excessive iron accumulation may reflect neurodegeneration. As shown in a clinical trial, iron chelation such as deferiprone can preserve behavioral motor functions and reduce iron concentration levels in the spinal cord, medulla oblongata, and motor cortex (33). This study supported the idea that iron deposition may be a novel therapeutic target for treatment.

In this study, we found that the QSM value was significantly correlated with the GM volume in the precentral gyrus and the thalamus in patients with ALS but not controls. Although evidence was lacking in ALS studies to show iron depositionGM atrophy coupling, a comparable result was presented in the multiple sclerosis (16) study where researchers found a significant association between iron deposition and reduction of GM volume in the left precentral gyrus. In this study, the significant relationship between iron deposition and GM volume in the precentral gyrus and the thalamus suggested that excessive iron content may contribute to brain structural atrophy. Since a large proportion of neurons in the thalamus relate to the motor cortex, iron deposition, as well as GM atrophy of the thalamus, may be attributed to the secondary effect after the impairment of neurons in the motor cortex. Since this study only offered a linear correlation between iron deposition and GM atrophy, it is, therefore, still a matter of debate whether iron deposition is a direct cause or a secondary event of structural impairment. The exact pathophysiological mechanism of ALS is multifactorial and requires further clarification.

The ALSFRS-R is a validated rating instrument for monitoring the progression of disability in patients with ALS, which correlates significantly with quality of life. The UMN score represents the severity of the UMN damage. In this study, we found that the QSM value of the precentral gyrus was significantly positively correlated with the UMN score, while the GM volume of the left precentral gyrus was negatively correlated with the UMN score. These observations indicate that iron deposition and GM atrophy in the precentral gyrus may together contribute to the UMN impairments. In addition to the precentral gyrus, the QSM value of the thalamus was significantly negatively correlated with the ALSFRS-R, suggesting that thalamic iron deposition is associated with ALS disease severity. The associations between thalamocortical iron deposition and the ALSFRS-R as well as the UMN score indicate strong clinical relevance. Specifically, iron deposition in the thalamus may affect the ability of sensorimotor information transmission and, therefore, patients with higher iron content in the thalamus exhibit more impaired overall physical functions as shown in the lower ALSFRS-R score. Based on these observations, we considered that the increased iron content in the thalamus may be an objective neuroimaging biomarker beyond the motor cortex that can reflect the disease severity of ALS. We did not obtain a significant correlation between the disease duration and the GM volume in the motor cortex. There are two potential reasons. First, the heterogeneity of the ALS in addition to the small sample size in this study may cause a negative finding. Second, we selected ROI in a post-hoc manner, which was based on the clusters that showed the most significant accumulation of iron content, which may not directly correlate most with the disease duration.

The major limitation of this study is the relatively small sample size, which limited the statistical power. As a result, the negative correlation between iron deposition and GM volume in the thalamus reached a one-tailed significance level. Although we have a prior hypothesis, the association between iron deposition and GM volume may become more significant with relatively larger sample size. In addition, although the QSM value correlates strongly with iron deposition 
in GM, it is an indirect measurement, which is difficult to mark the exact pathological process in the neurons. The third limitation of this study is the lack of postmortem examination so we cannot confirm the exact neurological underpinnings. From the methodological point of view, we applied the T1-normalization approach to normalizing the QSM images and found eight subjects that the images were not coregistered into the MNI template accurately. Future studies with a large sample size are encouraged to investigate a more appropriate approach for better normalization of the QSM images.

In conclusion, this study provided evidence of increased QSM values, decreased GM volume in the motor-thalamic loop in patients with ALS, supporting a probable association of iron deposition and GM atrophy as hallmarks of the disease. The current results may bring insights into the thalamic pathologic mechanism in ALS.

\section{DATA AVAILABILITY STATEMENT}

The original contributions presented in the study are included in the article/Supplementary Material, further inquiries can be directed to the corresponding author.

\section{ETHICS STATEMENT}

The studies involving human participants were reviewed and approved by Xuanwu Hospital. The patients/participants provided their written informed consent to participate in this study.

\section{REFERENCES}

1. Kiernan MC, Vucic S, Cheah BC, Turner MR, Eisen A, Hardiman O, et al. Amyotrophic lateral sclerosis. Lancet. (2011) 377:942-55. doi: 10.1016/S0140-6736(10)61156-7

2. van der Graaff MM, de Jong JM, Baas F, de Visser M. Upper motor neuron and extra-motor neuron involvement in amyotrophic lateral sclerosis: a clinical and brain imaging review. Neuromuscul Disord. (2009) 19:538. doi: 10.1016/j.nmd.2008.10.002

3. Christidi F, Karavasilis E, Rentzos M, Kelekis N, Evdokimidis I, Bede P. Clinical and radiological markers of extra-motor deficits in amyotrophic lateral sclerosis. Front Neurol. (2018) 9:1005. doi: 10.3389/fneur.2018.01005

4. Tu S, Menke RAL, Talbot K, Kiernan MC, Turner MR. Regional thalamic MRI as a marker of widespread cortical pathology and progressive frontotemporal involvement in amyotrophic lateral sclerosis. J Neurol Neurosurg Psychiatry. (2018) 89:1250-8. doi: 10.1136/jnnp-2018318625

5. Schönecker S, Neuhofer C, Otto M, Ludolph A, Kassubek J, Landwehrmeyer B, et al. Atrophy in the Thalamus But Not Cerebellum Is Specific for C9orf72 FTD and ALS Patients - An Atlas-Based Volumetric MRI Study. Front Aging Neurosci. (2018) 10:45. doi: 10.3389/fnagi.2018.00045

6. Verstraete E, Veldink JH, Hendrikse J, Schelhaas HJ, van den Heuvel MP, van den Berg LH. Structural MRI reveals cortical thinning in amyotrophic lateral sclerosis. J Neurol Neurosurg Psychiatry. (2012) 83:3838. doi: 10.1136/jnnp-2011-300909

7. Chen Z, Ma L. Grey matter volume changes over the whole brain in amyotrophic lateral sclerosis: a voxel-wise meta-analysis of voxel

\section{AUTHOR CONTRIBUTIONS}

QL contributed to the volunteer recruitment, experimental design, data collection, statistics, and manuscript preparation. $\mathrm{WZ}, \mathrm{XW}$, and YD contributed to the patient recruitment and clinical data collection. ZZ contributed to the data analysis and statistics. JL contributed to the conception, funding, study design, supervision, and manuscript preparation. All authors contributed to the article and approved the submitted version.

\section{FUNDING}

This study was supported by the National Natural Science Foundation of China $(82001352,81801255)$, the Capital Health Development Research Project (2018-4-2015), the Beijing Hospitals Authority Youth Program (QML20190803), and the Beijing Municipal Administration of Hospitals' Ascent Plan, code: DFL20180802.

\section{ACKNOWLEDGMENTS}

We would like to thank Dr Juan Wei from GE Healthcare, MR Research China, for technical support and also Dr Yi Wang from Cornell University for valuable discussion.

\section{SUPPLEMENTARY MATERIAL}

The Supplementary Material for this article can be found online at: https:/www.frontiersin.org/articles/10.3389/fneur. 2022.791300/full\#supplementary-material

based morphometry studies. Amyotroph Lateral Scler. (2010) 11:54954. doi: 10.3109/17482968.2010.516265

8. Menke RA, Körner S, Filippini N, Douaud G, Knight S, Talbot K, et al. Widespread grey matter pathology dominates the longitudinal cerebral MRI and clinical landscape of amyotrophic lateral sclerosis. Brain. (2014) 137(Pt 9):2546-55. doi: 10.1093/brain/awu162

9. Chipika RH, Siah WF, Shing SLH, Finegan E, McKenna MC, Christidi F, et al. MRI data confirm the selective involvement of thalamic and amygdalar nuclei in amyotrophic lateral sclerosis and primary lateral sclerosis. Data Brief. (2020) 32:106246. doi: 10.1016/j.dib.2020.106246

10. Kwan JY, Jeong SY, Van Gelderen P, Deng HX, Quezado MM, Danielian LE, et al. Iron accumulation in deep cortical layers accounts for MRI signal abnormalities in ALS: correlating 7 tesla MRI and pathology. PLoS ONE. (2012) 7:e35241. doi: 10.1371/journal.pone.0035241

11. Langkammer C, Enzinger C, Quasthoff S, Grafenauer P, Soellinger M, Fazekas F, et al. Mapping of iron deposition in conjunction with assessment of nerve fiber tract integrity in amyotrophic lateral sclerosis. J Magn Reson Imaging. (2010) 31:1339-45. doi: 10.1002/jmri.22185

12. Acosta-Cabronero J, Machts J, Schreiber S, Abdulla S, Kollewe K, Petri S, et al. Quantitative susceptibility MRI to detect brain iron in amyotrophic lateral sclerosis. Radiology. (2018) 289:195-203. doi: 10.1148/radiol.2018180112

13. Costagli M, Donatelli G, Biagi L, Caldarazzo Ienco E, Siciliano G, Tosetti $\mathrm{M}$, et al. Magnetic susceptibility in the deep layers of the primary motor cortex in amyotrophic lateral sclerosis. Neuroimage Clin. (2016) 12:9659. doi: 10.1016/j.nicl.2016.04.011

14. De Reuck JL, Deramecourt V, Auger F, Durieux N, Cordonnier C, Devos D, et al. Iron deposits in post-mortem brains of patients with neurodegenerative 
and cerebrovascular diseases: a semi-quantitative 7.0 T magnetic resonance imaging study. Eur J Neurol. (2014) 21:1026-31. doi: 10.1111/ene.12432

15. Ward RJ, Zucca FA, Duyn JH, Crichton RR, Zecca L. The role of iron in brain ageing and neurodegenerative disorders. Lancet Neurol. (2014) 13:104560. doi: 10.1016/S1474-4422(14)70117-6

16. Rumzan R, Wang JJ, Zeng C, Chen X, Li Y, Luo T, et al. Iron deposition in the precentral grey matter in patients with multiple sclerosis: a quantitative study using susceptibility-weighted imaging. Eur J Radiol. (2013) 82:e959. doi: 10.1016/j.ejrad.2012.09.006

17. Brooks BR, Miller RG, Swash M, Munsat TL. El Escorial revisited: revised criteria for the diagnosis of amyotrophic lateral sclerosis. Amyotroph Lateral Scler Other Motor Neuron Disord. (2000) 1:293-9. doi: 10.1080/146608200300079536

18. Cedarbaum JM, Stambler N, Malta E, Fuller C, Hilt D, Thurmond $\mathrm{B}$, et al. The ALSFRS-R: a revised ALS functional rating scale that incorporates assessments of respiratory function. J Neurol Sci. (1999) 169:1321. doi: 10.1016/S0022-510X(99)00210-5

19. Cosottini M, Donatelli G, Costagli M, Caldarazzo Ienco E, Frosini D, Pesaresi I, et al. High-Resolution 7T MR imaging of the motor cortex in amyotrophic lateral sclerosis. AJNR Am J Neuroradiol. (2016) 37:45561. doi: 10.3174/ajnr.A4562

20. Kimura F, Fujimura C, Ishida S, Nakajima H, Furutama D, Uehara H, et al. Progression rate of ALSFRS-R at time of diagnosis predicts survival time in ALS. Neurology. (2006) 66:265-7. doi: 10.1212/01.wnl.0000194316.91908.8a

21. Wei J, Wang L, Zhang J, Wei H, Zhang Y, Cheng X, et al., editors. Quantitative Susceptibility Mapping for Drug-Addicted Human Brain. In: 2018 14th IEEE International Conference on Signal Processing (ICSP); 2018. (2018). pp. 126. doi: 10.1109/ICSP.2018.8652449

22. Popescu V, Battaglini M, Hoogstrate WS, Verfaillie SC, Sluimer IC, van Schijndel RA, et al. Optimizing parameter choice for FSL-Brain Extraction Tool (BET) on 3D T1 images in multiple sclerosis. Neuroimage. (2012) 61:1484-94. doi: 10.1016/j.neuroimage.2012.03.074

23. Wei $\mathrm{H}$, Xie L, Dibb $\mathrm{R}$, Li W, Decker $\mathrm{K}$, Zhang $\mathrm{Y}$, et al. Imaging whole-brain cytoarchitecture of mouse with MRIbased quantitative susceptibility mapping. Neuroimage. (2016) 137:107-15. doi: 10.1016/j.neuroimage.2016.05.033

24. Wei H, Dibb R, Zhou Y, Sun Y, Xu J, Wang N, et al. Streaking artifact reduction for quantitative susceptibility mapping of sources with large dynamic range. NMR Biomed. (2015) 28:1294-303. doi: 10.1002/nbm.3383

25. Langkammer C, Schweser F, Krebs N, Deistung A, Goessler W, Scheurer E, et al. Quantitative susceptibility mapping (QSM) as a means to measure brain iron? A post mortem validation study. NeuroImage. (2012) 62:15939. doi: 10.1016/j.neuroimage.2012.05.049
26. Ashburner J. A fast diffeomorphic image registration algorithm. Neuroimage. (2007) 38:95-113. doi: 10.1016/j.neuroimage.2007.07.007

27. Rolls ET, Huang CC, Lin CP, Feng J, Joliot M. Automated anatomical labelling atlas 3. Neuroimage. (2020) 206:116189. doi: 10.1016/j.neuroimage.2019.116189

28. Hwang K, Bertolero MA, Liu WB, D'Esposito M. The human thalamus is an integrative hub for functional brain networks. J Neurosci. (2017) 37:5594607. doi: 10.1523/JNEUROSCI.0067-17.2017

29. Fan L, Li H, Zhuo J, Zhang Y, Wang J, Chen L, et al. The human brainnetome atlas: a new brain atlas based on connectional architecture. Cereb Cortex. (2016) 26:3508-26. doi: 10.1093/cercor/bhw157

30. Lovejoy DB, Guillemin GJ. The potential for transition metal-mediated neurodegeneration in amyotrophic lateral sclerosis. Front Aging Neurosci. (2014) 6:173. doi: 10.3389/fnagi.2014.00173

31. $\mathrm{Bu} \mathrm{XL}$, Xiang $\mathrm{Y}$, Guo Y. The role of iron in amyotrophic lateral sclerosis. Adv Exp Med Biol. (2019) 1173:145-52. doi: 10.1007/978-981-13-95 89-5_8

32. Lee JK, Shin JH, Gwag BJ, Choi EJ. Iron accumulation promotes TACEmediated TNF- $\alpha$ secretion and neurodegeneration in a mouse model of ALS. Neurobiol Dis. (2015) 80:63-9. doi: 10.1016/j.nbd.2015.05.009

33. Moreau C, Danel V, Devedjian JC, Grolez G, Timmerman K, Laloux $\mathrm{C}$, et al. Could conservative iron chelation lead to neuroprotection in amyotrophic lateral sclerosis? Antioxid Redox Signal. (2018) 29:7428. doi: 10.1089/ars.2017.7493

Conflict of Interest: The authors declare that the research was conducted in the absence of any commercial or financial relationships that could be construed as a potential conflict of interest.

Publisher's Note: All claims expressed in this article are solely those of the authors and do not necessarily represent those of their affiliated organizations, or those of the publisher, the editors and the reviewers. Any product that may be evaluated in this article, or claim that may be made by its manufacturer, is not guaranteed or endorsed by the publisher.

Copyright (C) $2022 \mathrm{Li}, \mathrm{Zhu}, \mathrm{Wen}, \mathrm{Zang}, \mathrm{Da}$ and Lu. This is an open-access article distributed under the terms of the Creative Commons Attribution License (CC BY). The use, distribution or reproduction in other forums is permitted, provided the original author(s) and the copyright owner(s) are credited and that the original publication in this journal is cited, in accordance with accepted academic practice. No use, distribution or reproduction is permitted which does not comply with these terms. 